\title{
Comparison of applications used to help the elderly
}

\author{
Liza Privošnik, Maša Sedej Knezović, Lara Šinkovec, Andrej Starc \\ University of Ljubljana, Faculty of Health Sciences, Ljubljana, Slovenia
}

\section{Abstract}

Introduction: The COVID-19 pandemic has radically changed the existing infrastructure of medical practice, as clinics and hospitals around the world have focused on virtual resources to help care for patients. Technology has become critical to our wellbeing. Specifically, the technology for older adults is addressed for content that the elderly have the most needs in. The aim of this literature review is to present a comparison of mobile applications designed to help older adults in their basic life activities. Methods: A quantitative descriptive research method with critical review of English scientific and professional literature was performed using the following databases DiKul, PubMed, Researchgate and SienceDirect. The literature search took place from March 2021 to April 2021. Literature inclusion criteria were articles published between 2016 and 2021, open and free access and articles with clearly defined objectives and methods in English language. The exclusion criteria were articles published before 2016 , articles with paid access and articles that were not in English language. We reviewed 51 articles and excluded 35 articles that didn't match our criteria. We included 16 articles. Data was analyzed using a content analysis method. Results: The results showed that there are a few different mobile applications for different conditions in the market. All of them are designed to make life easier for elderly. There is evidence that mobile applications are helping elderly population as much as they help health workers. The benefits of reviewed mobile applications are to help reduce rates of forgetting and of medication errors, and also increases perceived independence in managing medication. However, currently available applications for smart mobile terminals used by the aged, which serve as the important carrier of various internet services for elderly people, have obvious defects in quantity, service range, and charm. Results show that in any mobile application that we included in this review there is room for 
improvement. Discussion and conclusions: It is important to bridge the gap between the elderly people and modern technologies, which is achieved through more products designed based on emotional care and construction, and by providing an appropriate training program for the elderly population to use a smartphone. In the future, research should focus on testing mobile health interventions in patients at risk for poor adherence and on standardizing alerts and protocols for clinicians.

Keywords: elderly, technology, mobile application, ICT

\section{Introduction}

The COVID-19 pandemic has radically changed the existing infrastructure of medical practice, as clinics and hospitals around the world have focused on virtual resources to help care for patients. Various professional associations emphasize the importance of the transition to telemedicine whenever possible (Lakkireddy et al., 2020). Technology has become critical to our wellbeing. Technology also presents many opportunities for expanding the possibilities of having a good life, and consequently is now somehow a necessity (Uysal et al., 2016).

The interest and needs of older adults are mainly focused around employment, housing, health and social care (Özsungur, 2019). Specifically, the technology for older adults is addressed for content, that the elderly have the most needs in. That includes programs and mobile applications for healthcare monitoring needs (medication instruction), social needs, and also safety and privacy needs (Klimova, Karesova, 2016).

The aim of this literature review is to present a comparison of mobile applications designed to help older adults in their basic life activities.

\section{Methods}

A quantitative descriptive research method with critical review of English scientific and professional literature was performed using the following databases DiKul, PubMed, Researchgate and SienceDirect. The keywords we used for searching literature were: elderly, technology, mobile application and ICT. Literature inclusion criteria were articles published between 2016 and 2021, open and free access and articles with clearly defined objectives and methods in English language. We reviewed 51 articles and excluded 35 articles that didn't match our criteria. We included 16 articles. The literature search took place in March 2021. Data was analysed using a content analysis method.

\section{Results}

The results of the review are presented in Table 1, where the first author and year of publication, methods and main results are written. 
A preliminary analysis was made by in-

Qi, 2016 terviewing 20 elderly people between $60-70$ years old on their usage of the mobile applications.

181 patients with diverse cancers who were prescribed oral therapy were randomized to receive either the smartphone mobile application or stand-

Greer, 2020 ard care. Linear regression was used to assess intervention effects on adherence and change in self-report outcomes from baseline to week 12, controlling for baseline scores and social support.

This retrospective cohort study included patients with diabetes recruited to the Lilly Connected Care Program (LCCP) platform for 8 months. According to the number of diabetes education courses they had completed, the patients were divided into three groups. Univariate and multivariate linear regression analyses were used.
Currently available mobile applications for smart mobile terminal used by the aged, which serve as the important carrier of various defects in quantity, service, range and charm.

Although the mobile application may not improve outcomes for all patients prescribed oral cancer therapy, the intervention may be beneficial for those with certain risk factors, such as difficulties with adherence or anxiety.

LCCP application-based diabetes education is effective for glycemic control and SMBG behavior improvement in patients with type 2 diabetes receiving insulin therapy.
41 participants performed three CMJs assessed via a contact mat and the My Jump application. The intraclass corre-

Cruvinel-Cabral, 2018 lation coefficient was used to verify the relative reliability, the coefficient of variation and the typical error of measurement were used to verify the absolute reliability.

The aim was to determine whether a prevention mobile application "Aachen fall prevention App" (AFPA) would be accepted and downloaded voluntarily by persons in the target population.

A quasi-experimental design was employed. Seventeen older people self-reported health concerns via Interaktor twice a week for 3-months and answered questionnaires at baseline, the end of the intervention and at a 6 -month follow-up.

Authors conducted semi-structured interviews in two groups to uncover their recommendations and requirements of application, the one was patients who never used applications that related to diabetes and another group was patients who ever used it.
Results suggest that the My Jump application is a valid and reliable tool compared to the contact mat for evaluating vertical jump performance in the elderly.

This field study revealed the AFPA as a promising tool to raise older adults awareness of their individual fall risk by means of a low-threshold patient-driven fall risk assessment tool.

The high application usage showed that an application may be a suitable tool for some older people living alone and receiving home care. The results indicate that the usage of Interaktor can support older people by significantly improving their communicative and critical health literacy.

A total of 24 empty nest elderly were interviewed, among them, 11 patients use smartphone and 13 not. Four themes were formed including knowledge of diabetes, record and reminders of medicine taking, blood glucose tests, appointments, communicate with peers and health service providers, feedback from health service providers. 
Researchers wanted to investigate to what extent two-week device-supported cycle training (modified version of the THERA-Trainer tigo ${ }^{\circ}$ and application-based fall risk assessment (certified medical application Lindera Mobilitätsanalyse) are feasible measures for fall prevention in older people living at

Fuchs, 2020 home. They used one-group pre-post design over a two-week intervention period. A questionnaire assessed participants'motivation, and a fall risk score was calculated pre- and post-intervention. The qualitative interviews were carried out retrospectively among two expert service providers after the intervention study.

Zhao, 2020

Zhong, 2020

Isaković, 2016 wait-list control group or the intervention group according to a random number. A smartphone training program was developed and was then made up of two modules. The first training module was easiest and focused on theoretical aspects. The second was focused on practical aspects, covering various smartphone applications.

A single-blind randomized controlled trial was conducted with a control and an experimental group in Spain in 2013. The characteristics of application called ALICE were specified based on the suggestions of 3 nominal groups with a total of 23 patients and a focus group with 7 professionals.

Authors recruited a total of 148 community-dwelling older adults aged 60 years and older from two cities in China: Beijing and Chongqing. User acceptability was measured by a questionnaire including four quantitative measures.

A nonequivalent control group with a non-synchronized design was utilized, and 60 participants were recruited from Chungnam National University Hospital from March to August 2018. Data were analysed using the $\chi^{2}$-test, the t-test, the repeated-measures ANOVA, and the $\mathrm{McNemar}$ test.

Authors perform subsequent usability evaluations on end users alone. The users were all older than 50 , with the average age of 64.2 years.
344 participants were included. The participants were randomly assigned to the
The results showed a high subjective motivation during the training and a slight reduction of the fall risk. Both the training and the assessment are feasible and well-received interventions. The respondents' self-assessed affinity for technology was moderate.

A significant improvement in smartphone usage competency for participants in the intervention group compared with the wait-list control group was reported. In the intervention group was presented a significant increase in smartphone application proficiency. However, that did not occur in wait-list control group. The intervention group showed big improvement in quality of life compared to the wait-list control group.

The ALICE application improves adherence, helps reduce rates of forgetting and of medication errors, and increases perceived independence in managing medication. Also elderly were able to use this application without any knowledge of computer skills.

This mobile phone application is a health management tool for older adults to self-manage their gait quality and prevent adverse outcomes.

The mobile- application-based self-management program developed in this study increased the sick-role behavior, basic psychological needs, and self-efficacy of elderly hemodialysis patients, while physiological parameters were maintained within the normal range.

The results show, that applications developed for the general population are not necessarily suitable for elderly users, which can be a significant problem, especially if they address the issues of the elderly users specifically. 
This study was a nonprobabilistic pilot trial using convenience sampling. An intervention was implemented among a group of 22 older adults between 60

Martínez-Alcalá, 2018 and 80 years of age over 12 weeks. Half of the older adults were stimulated with the mobile application and the other half followed the traditional paper and pencil training.

A randomized controlled study was conducted in subjects aged 6o years and older with food insecurity, identified at 17

Rodrigues, 2017 primary care centers in the Lisboa e Vale do Tejo health region in Lisbon, Portugal. The primary outcome was the changes in participants' food insecurity score at 3 months.

The subjects of the study were elderly people who wanted to check their weight and cardiac status. For this purpose, two mobile applications were used to measure energy expenditure based on physical activity and heart rate during controlled walking at specific speeds.
The intervention data show that the experimental group obtained better results in the postevaluation given that the participants were able to execute the exercises repetitively.

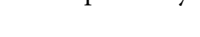

This study assesses the efficacy of this innovative tool for disseminating relevant health information, modifying behaviors, and decreasing food insecurity in an easy, low-cost, and massive way.

\section{Discussion}

In this information era, mobile applications have become an important tool in our work, life and entertainment. Third-party mobile applications that provide us with various convenient services have gradually become an essential part of our daily lives. Elderly people have been gradually becoming an important strength supporting the internet economy. However, currently available mobile applications for smart mobile terminals used by the aged, which serve as the important carrier of various internet services for elderly people, have obvious defects in quantity, service range, and charm (Qi et al., 2016). As Qi et al. (2016) point out, bridging the gap between elderly people and modern technologies requires more products designed based on emotional care and construction. Fields such as the design of intelligent mobile terminals and mobile applications for elderly still need further development.

It is important for creators that are developing mobile application used by the elderly people, to be aware of the shortcomings and problems that the elderly people face when using previously mentioned applications. If the mobile application is created well, used by in our case elderly people, helps reduce rates of forgetting and of medication errors, can also increase perceived independence in managing medication, reduction of fall risk, and also improving their communicative and critical health literacy.

An analysis of the mobile application for diabetes DeStress Assistant (DeSA), which was developed within the EU project and tested in a hospital by Isaković and colleagues (2016), showed that with a limited amount of modi- 
fications, an existing mobile application can be significantly improved to better suit elderly users. This could also be facilitated by creating different profiles to optimize the mobile application for different accessibility groups (e.g., poor eyesight and limited dexterity). A user could simply select their profile and the mobile application would be configured to their preferences. Such personalization features would of course have significantly larger impact and reach, if they were consistently implemented in all of the major mobile device operating systems.

When creating a mobile application designed for older adults with diabetes, creators need to focus on four important points: 1) The patient's knowledge of diabetes; 2) Record and reminders of medicine taking, blood glucose test, appointments; 3) Communicate with peers and health service providers; 4) Feedback from health service providers (Lv et al., 2018). In view of all the above, the mobile applications in question can also help healthcare professionals. Patients would not need frequent doctor's appointment, as only through the application used by their patients, could they find out how well the patient knows and works within the aforementioned four points.

Fall incidents are also a major problem for elderly patients and also healthcare. The Aachen Fall Prevention application (AFPA) represents the first mobile Health application empowering older patients (persons 50+ years) to self assess and monitor their individual fall risk. Rosche et al. (2017) conducted a study that revealed that AFPA is a promising tool to raise older adults' awareness of their individual fall risk by means of a low-threshold patient-driven fall risk assessment tool.

\section{Conclusions}

Mobile applications have become an important tool of our everyday life. There are a few different mobile applications for different conditions on the market. All of them are designed to make life easier for elderly. It is important to bridge the gap between the elderly people and modern technologies, which is achieved through more products designed based on emotional care and construction, and by providing an appropriate training program for the elderly population to use a smartphone.

Well-created mobile applications help reduce rates of forgetting and of medication errors, can also increase perceived independence in managing medication, reduction of fall risk, it improves knowledge about their disease and how to control it, and also improving their communicative and critical health literacy.

In the future, research should focus on testing mobile health interventions in patients at risk for poor adherence and on standardizing alerts and protocols for clinicians. 


\section{References}

BOUKHENNOUFA, I., AMIRA, A., BENSAALI, F. and ESFAHANI, S.S., 2020. A novel gateway-based solution for remote elderly monitoring. Journal of biomedical informatics, vol. 109, pp. 1-11. Available from: http://dx.doi. org/10.1016/j.jbi.2O20.103521

CRUVINEL-CABRAL, R.A., OLIVEIRA-SILVA, I., RICARTE MEDEIROS, A., CLAUDINO, J.G., JIMÉNEZ-REYES, P. and BOULLOSA, D.A., 2018. The validity and reliability of the "My Jump App" for measuring jump height of the elderly. Peer J, vol. 6, pp. e5804. Available from: https://doi. org/10.7717/peerj.5804

DAMANT, J. and KNAPP, M., 2015. What are the likely changes in society and technology which will impact upon the ability of older adults to maintain social (extra-familial) networks of support now, in 2025 and in 2040 ? London, 2015 [online]. [viewed 10 April 2021]. Available from: https:// core.ac.uk/download/pdf/35436349.pdf

FUCHS, D., TIEBEL, J. and FRIEDRICH, P., 2020. Device-supported training and assessment for fall prevention of community-dwelling elderly: a pre-post mixed methods study. Procedia Computer Science, vol. 176, pp. 2322-31. Available from: https://doi.org/10.1016/j.procs.2020.09.293

GÖRANSSON, C., WENGSTRÖM, Y., HÄLLEBERG-NYMAN, M., LANGIUS-EKLÖF, A., ZIEGERT, K. and BLOMBERG, K., 2020. An app for supporting older people receiving home care - usage, aspects of health and health literacy: a quasiexperimental study. BMC Medical Informatics and Decision Making, vol. 20, no. 1, pp. 226. Available from: https://doi. org/10.1186/s12911-02O-01246-3

GREER, J.A., JACOBS, J.M., PENSAK, N., NISTOEL, L.E., FISHBEIN, J.N., MACDONALD, J.J., REAM, M., WALSH, E.A., BUZAGO, J., MUZIKANSKY, A., et al., 2020. Randomized trial of a smartphone mobile app to improve symptoms and adherence to oral therapy for cancer. Journal of the National Comperhensive Cancer Network, vol. 18, no. 2., pp. 133-41. Available from: https://doi.org/10.6004/jnccn.2019.7354

IANCU, I. and IANCU, B., 2020. Designing mobile technology for elderly. A theoretical overview. Tehnological Forecasting and Social Change, vol. 155, pp. 1-9. Available from: https://doi.org/10.1016/j.techfore.2020.119977

ISAKOVIĆ, M., SEDLAR, U., VOLK, M. and BEŠTER, J., 2016. Usability pitfalls of diabetes mHealth apps for the elderly. Journal of diabetes research, vol. 2016, no. 2, pp. 1-9. Available from: http://dx.doi.org/10.1155/2016/1604609

KLIMOVA, B., and MARESOVA, P., 2016. Elderly people and their attitude towards mobile phones and their applications - a review study. In: PARK. J.H., CHAO, H.C., ARABNIA, H.R., YEN, N.Y, ed. Advanced Multimedia and Ubiquitous Engineering [online]. New York: Springer. [viewed 10 March 2021]. Available from: 10.1007/978-3-662-47487-7 
LAKKIREDDY, D.R., CHUNG, M.K., GOPINATHANNAIR, R., PATTON, K.K., GLUCKMAN, T.J., TURAGAM, M., CHEUNG, J.W., PATEL, P., SOTOMONTE, J., LAMPERT, R., HAN, J.K., et al., 2020. Guidance for cardiac electrophysiology during the COVID-19 pandemic from the Heart Rhythm Society COVID-19 Task Force; Electrophysiology Section of the American College of Cardiology; and the Electrocardiography and Arrhythmias Committee of the Council on Clinical Cardiology, American Heart Association. Hearth Rhythm Society, vol. 17, no. 9, pp. e233e241. Available from: https://doi.org/10.1016/j.hrthm.2020.03.028

LV, X., XIA, J., FU, L. and CAO, Y., 2018. Design of a mobile APP for empty nest elderly patients with T2DM. Studies in health technology and informatic, vol. 250, pp. 146-147. [viewed 10 April 2021]. Available from: https://pubmed.ncbi.nlm.nih.gov/29857411/

MARTÍNEZ-ALCALÁ, C.I., ROSALES-LAGARDE, A., HERNÁNDEZ-ALONSO, E., MELCHOR-AGUSTIN, R., RODRIGUEZ-TORRES, E.E. and ITZÁ-ORTIZ, B.A., 2018. A mobile app (iBeni) with a neuropsychological basis for cognitive stimulation for elderly adults: pilot and validation study. Journal of medical internet research, research protocols, vol. 7, no. 8. Available from: http://dx.doi.org/10.2196/resprot.9603

MIN, Y. and PARK, M., 2020. Effects of a mobile-app-based self-management support program for elderly hemodialysis patients. Healthcare informatics research, vol. 26, no. 2, pp. 93-103. Available from: http://dx.doi. org/10.4258/hir.2020.26.2.93

MIRA, J.J., NAVARRO, I., BOTELLA, F., BORRÁS, F., NUÑO-SOLINÍS, R., OROZCO, D., IGLESIAS-ALONSO, F., PÉREZ-PÉREZ, P., LORENZO, S. and TORO, N., 2014. A Spanish pillbox app for elderly patients taking multiple medications: randomized controlled trial. Journal of medical internet research, vol. 16, no. 4. Available from: http://dx.doi.org/10.2196/ jmir.3269

ÖZSUNGUR, F., 2019. Gerontechnological factors affecting successful aging of elderly. The aging male, vol. 23 , no. 5, pp. 520-532. Available from: http:// dx.doi.org/10.1080/13685538.2018.1539963

QI, X., ZHANG, W. and HAO, F., 2016. Design of emotional constructionBased life service APP for elderly people. 2016 Intl IEEE Conferences on Ubiquitous Intelligence \& Computing, Advanced and Trusted Computing, Scalable Computing and Communications, Cloud and Big Data Computing, Internet of People, and Smart World Congress (UIC/ ATC/ScalCom/CBDCom/IoP/SmartWorld), Toulouse, France 18.- 22. July 2016 [online]. Piscataway: IEEE pp. 1045-51. Available from: https://doi. org/10.1109/UIC-ATC-ScalCom-CBDCom-IoP-SmartWorld.2016.0163

RAMIREZ LOPEZ, L.J., GUILLEN PINTO, E.P. and RAMOS LINARES, C.O., 2018. Effective validation model and use of mobile-health applications for the elderly. Healthcare informatics research, vol. 24, no. 4, pp. 276-282. Available from: http://dx.doi.org/10.4258/hir.2018.24.4.276 
RASCHE, P., MERTENS, A., BROHL, C., THEIS, S., SEINSCH, T., WILLE, M., PAPE, H.C. and KNOBE, M., 2017. The "Aachen fall prevention App" - a Smartphone application app for the self assessment of elderly patients at risk for ground level falls. Patient Safety in Surgery, vol. 11, no. 14. Available from: https://doi.org/10.1186/s13037-017-0130-4

RODRIGUES, A.M., GREGÓRIO, M.J., GEIN, P., EUSÉBIO, M., SANTOS, M.J., DE SOUSA, R.D., COELHO, P.S., MENDES, J.M., GRAÇA, P., OLIVEIRA, P., BRANCO, J.C. and CANHÃO, H., 2017. Home-based intervention program to reduce food insecurity in elderly populations using a TV app: study protocol of the randomized controlled trial Saúde. Come Senior. Journal of medical internet research, research protocols, vol. 6, no. 3. Available from: http://dx.doi.org/10.2196/resprot.6626

UYSAL, M., SIRGY, M.J., WOO, E. and KIM, H.L., 2016. Quality of life (QOL) and well-being research in tourism. Tourism management, vol. 53, pp. 244-261. Available from: http://dx.doi.org/10.1016/j.tourman.2015.07.013

ZHANG, Y., LIU, C., LOU, S., HUANG, J., LI, X. and ZHOU, Z., 2020. Effectiveness of Lilly Connected Care Program (LCCP) App-Based Diabetes Education for Patients With Type 2 Diabetes Treated With Insulin: Retrospective Real-World Study. JMIR Mhealth and Uhealth, vol. 8, no. 3, pp. e17455. Available from: https://doi.org/10.2196/17455

ZHAO, X., WANG, L., GE, C., ZHEN, X., CHEN, Z., WANG, J. and ZHOU, Y., 2020. Smartphone application training program improves smartphone usage competency and quality of life among the elderly in an elder university in China: A randomized controlled trial. International journal of medical informatics, vol. 133, pp. 1-8. Available from: http://dx.doi. org/10.1016/j.ijmedinf.2019.104010

ZHONG, R. and RAU, P.P., 2020. A mobile phone-based gait assessment app for the elderly: development and evaluation. Journal of medical internet research, Mhealth and Uhealth, vol. 8, no. 5. Available from: http://dx.doi. org/10.2196/14453 